\title{
Research paper \\ EF-Tu from the enacyloxin producing Frateuria W-315 strain: Structure/activity relationship and antibiotic resistance
}

\author{
Jean-Bernard Créchet ${ }^{a,}{ }^{*}$, Christian Malosse ${ }^{\mathrm{b}}$, Codjo Hountondji ${ }^{\mathrm{c}}$ \\ ${ }^{a}$ Ecole Polytechnique, Route de Saclay, F-91120 Palaiseau, France \\ ${ }^{\mathrm{b}}$ Institut Pasteur, Département de Biologie Structurale et Chimie, Unité Spectrométrie de Masse Structurale et Protéomique, CNRS UMR 3528, \\ 28 rue du Dr Roux, 75724 PARIS Cedex 15 France \\ ' Sorbonne Universités UPMC Univ Paris 06, Unité de Recherche UPMC UR6 “Enzymologie de l'ARN”, 4, Place Jussieu, F-75252 Paris Cedex 05, France
}

Keywords:

Frateuria

Elongation factor Tu

Antibiotics resistance

\begin{abstract}
A B S T R A C T
In this report, we have demonstrated that the poly(U)-dependent poly(Phe) synthesis activity of elongator factor Tu (EF-Tu) from the enacyloxin producing strain Frateuria sp. W-315 is inhibited by the antibiotic similarly to that of Escherichia coli EF-Tu. The inhibitory effect of enacyloxin observed in a purified system was the same as that obtained with an S30 extract from E. coli or Frateuria sp. W-315, respectively, suggesting that antibiotic resistance of enacyloxin producing Frateuria sp. W-315 is not due neither to EF-Tu nor to other components of the translation machinery but to a still unknown mechanism. The EF-Tu gene, as PCR amplified from Frateuria W-315 genomic DNA and sequenced represented an ORF of 1191 nucleotides corresponding to 396 amino acids. This protein is larger than the product of tufA from E. coli by only two amino acid residues. Alignment of the amino acid sequence of EF-Tu from E. coli with those of Frateuria and Ralstonia solanacearum indicates on average $80 \%$ identical amino acid residues and 9.7\% conservative replacements between EF-Tu Frateuria and EF-Tu E. coli, on one hand, and 97\% identity and 1.7\% conservative replacement between EF-Tu Frateuria and EF-Tu Ralstonia solanacearum, on the other hand. These strong primary structure similarities between EF-Tu from different origins are consistent with the fact that this factor is essential for the translation process in all kingdoms of life. Comparison of the effects of antibiotics on EF-Tu Frateuria and EF-Tu E. coli revealed that enacyloxin, kirromycin and pulvomycin exert a stronger stimulation of the GDP dissociation rate on EFTu Frateuria, while the effects of the antibiotics on the GDP association rate were comparable for the two EF-Tu species. Different mutants of EF-Tu E. coli were constructed with the help of site directed mutagenesis by changing one or several residues of EF-Tu E. coli by the corresponding residues of EF-Tu Frateuria. The single A45K substitution did not modify the intrinsic GTPase activity of EF-Tu E. coli. In contrast, a 2-3 fold stimulation of the intrinsic GTPase activity was observed with the single A42E, F46Y, Q48E and the double F46Y/Q48E substitution. Finally, up to a 7 fold stimulation was observed with the quadruple substitution (mutant A42E/A45K/F46Y/Q48E.
\end{abstract}

\section{Introduction}

In all kingdoms of life, protein biosynthesis is promoted by two ribosomal subunits of unequal size (the small size subunit is $30 \mathrm{~S}$ and $40 \mathrm{~S}$, and the large size subunit is 50S and 60S, in bacteria and in eukaryotes, respectively) with the help of a number of translation factors (reviewed in Ref. [1]. Among these factors, several such as

\footnotetext{
* Corresponding author.

E-mail address: jean-bernard.crechet@polytechnique.edu (J.-B. Créchet).
}

initiation factor 2 (IF2), elongation factors Tu (EF-Tu) and G (EF-G) and release factors 3 (RF3 and eRF3) are GTPases. EF-Tu forms a stable ternary complex with aminoacyl-tRNA (aa-tRNA) and GTP and this complex interacts with the ribosome and delivers aa-tRNA to the A-site [2]. Aa-tRNA delivery to the ribosomal A-site comprises several steps: (i) initial binding of the ternary complex to the ribosome is codon-independent; (ii) interaction between the anticodon of aa-tRNA and the A-site mRNA codon insures the correct positioning of the aa-tRNA prior to peptide bond formation at the ribosomal peptidyl transferase center (PTC). In fact, formation of a correct codon-anticodon duplex induces a dramatic 
conformational change in the decoding site resulting in stabilization of the ternary complex on the ribosome. Furthermore, cognate codon recognition provides an activation signal that reaches the GTP-binding domain of EF-Tu and facilitates the formation of the activated GTPase state of the factor and the subsequent hydrolysis of the $\gamma$-phosphate of GTP; (iii) following the release of inorganic phosphate, the conformation of EF-Tu switches from the GTP to the GDP form which exhibits a low affinity for aa-tRNA. As a consequence, aa-tRNA is released from EF-Tu-GDP and is accommodated in the ribosomal 50S A-site in order to take part in peptide bond formation, while EF-Tu-GDP leaves the ribosome [3].

Owing to the essential role of translation in the rapid growth of pathogens sustaining bacterial infection, both $30 \mathrm{~S}$ and $50 \mathrm{~S}$ bacterial ribosomal subunits, as well as the translation factors are considered as valuable targets exhibiting several binding pockets for antibiotics, as deduced from the high-resolution structures of bacterial ribosomal subunits and those of their complexes with antibiotics $[4,5]$. Therefore, the discovery and development of new antibacterial agents has become a major topic in the post-crystal-structure era of the ribosome. In this context, an antibiotic consisting of a mixture of chemically related compounds was found to be produced by a bacterial Frateuria sp. W-315 strain. Frateuria W-315 strain has characteristics similar to those of Gluconobacter and produces a family of new polyenic antibiotics named enacyloxins which are active against Gram-negative and Gram-positive bacteria, only slightly active against fungi and not at all against yeast [6-8]. One of these compounds is enacyloxin IIa which was shown to inhibit bacterial protein biosynthesis in vitro [7]. EF-Tu was shown to be the specific target of enacyloxin [9]. EF-Tu is the target of three other families of antibiotics of unrelated structures [10,11], the prototypes of which are kirromycin, pulvomycin, and GE2270A. Enacyloxin IIa as kirromycin hinders the release of EF-Tu $\bullet$ GDP from the ribosome after GTP hydrolysis, thus inhibiting its recycling and peptide bound formation [9] while pulvomycin and GE2270A hinder the formation of the ternary complex between EF-Tu•GTP and aa-tRNA [12-14]. EF-Tu folds into three distinct domains, domain 1 being the nucleotide binding domain. Recent X-ray studies have revealed the tridimensional structures of EF-Tuantibiotics complexes. In one of these studies, kirromycin and enacyloxin were shown to share the same binding site located at the interface between domains 1 and $3[15,16]$. Pulvomycin was shown to contact all three domains, while GEA2270 was bound to domains 1 and 2 [15].

In the present report, we analyze the primary structure differences between EF-Tu from E. coli and EF-Tu from Frateuria sp. W 315 , after having cloned and sequenced the gene coding for this factor. The biochemical characterization of EF-Tu Frateuria, followed by comparison with EF-Tu Escherichia coli in regard to the interaction with guanine nucleotides, the GTPase activity, the effects of antibiotics (specially enacyloxin) and the mutations in the effector region provide key insights into the structure/activity relationship of EF-Tu.

Another question that we have addressed in the present report is the search for key resistance mutations in the amino acid sequence of EF-Tu from Frateuria sp-315 (as compared with the one of $E$. coli EF-Tu) that might be responsible for the resistance to the antibiotic of this enacyloxin producing Frateuria strain.

\section{Materials and methods}

\subsection{Purification of EF-Tu from Frateuria $W-315$}

$25 \mathrm{~g}$ of Frateuria W-315 cells obtained after $24 \mathrm{~h}$ at $25^{\circ} \mathrm{C}$ from $2 \mathrm{~L}$ culture in LB medium were sonicated 12 times for $10 \mathrm{~s}$ at $4{ }^{\circ} \mathrm{C}$ in lysis buffer containing $50 \mathrm{mM}$ Tris- $\mathrm{HCl} \mathrm{pH} 7.5,0.2 \mathrm{M} \mathrm{NaCl}, 5 \mathrm{mM}$
$\mathrm{MgCl}_{2}, 5 \mathrm{mM} \beta$-ME, $20 \mu \mathrm{M}$ GDP, $0.2 \mathrm{mg} \mathrm{ml}^{-1}$ lysosyme, $20 \mu \mathrm{g} \mathrm{ml}^{-1}$ DNase, one tablet of complete mini EDTA free protease inhibitor cocktail (Roche Diagnostics), and centrifuged (30 $000 \mathrm{~g}$ for $30 \mathrm{~min}$ ). The obtained supernatant which constituted S30 extract was centrifuged again at $100.000 \mathrm{~g}$ for $1 \mathrm{~h}$. Supernatant was dialysed against buffer A (25 mM Tris- $\mathrm{HCl}$ pH 7.5, $5 \mathrm{mM} \mathrm{MgCl} 2,30 \mathrm{mM} \mathrm{KCl}$, $7 \mathrm{mM} \beta$-ME, $20 \mu \mathrm{M}$ GDP), then loaded on Hi-Prep Source $30 \mathrm{Q}$ $16 \times 10$ (Äkta purifier system, GE Healthcare) using a linear $50-500 \mathrm{mM} \mathrm{KCl}$ gradient $(700 \mathrm{ml})$ in the same buffer. The active EF-Tu containing fractions (determined as $\left[{ }^{3} \mathrm{H}\right] \mathrm{GDP}$ binding on nitrocellulose filters) eluted between 150 and $200 \mathrm{mM} \mathrm{KCl}$ were concentrated by ultrafiltration, dialysed against buffer $\mathrm{A}$ and applied on Mono-Q HR5/5 (Äkta purifier system, GE Healthcare) with a 40 column volumes $50-300 \mathrm{mM} \mathrm{KCl}$ linear gradient in buffer A. The pooled and concentrated EF-Tu containing fractions (13 mg) were loaded on superdex $75(16 \times 60)$ GE Healthcare equilibrated in buffer A containing $0.2 \mathrm{M} \mathrm{KCl}$. The most pure fractions $(5 \mathrm{mg}$ ) as revealed on PAGE-SDS were collected, concentrated, dialysed against buffer A containing 50\% glycerol and stored at $-25{ }^{\circ} \mathrm{C}$.

\subsection{Determination of the amino acid sequence of tryptic peptides from purified Frateuria $W-315$ EF-Tu by mass spectrometry}

\subsubsection{Trypsin digestion}

Sample in $\mathrm{H}_{2} \mathrm{O}$ was reduced by one fifth volume of $10 \mathrm{mM}$ dithiothretiol (Sigma Aldrich) in $0.1 \mathrm{M} \mathrm{NH}_{4} \mathrm{HCO}_{3}$ for 30 min at $56{ }^{\circ} \mathrm{C}$ under agitation and alkylated by $55 \mathrm{mM}$ iodoacetamide in $0.1 \mathrm{M}$ $\mathrm{NH}_{4} \mathrm{HCO}_{3}$. The carbamidomethylated protein was digested with trypsin (Roche) in a 1:35 protein:trypsin ratio overnight at $37{ }^{\circ} \mathrm{C}$ under agitation. Sample was taken directly from the digest and desalted by C18 ZipTip ${ }^{\circledR}$ (Millipore) before MS analysis in 75:25:0.1 acetonitrile/water/HCOOH (v/v/v). Protein solution was desalted by C4 ZipTip ${ }^{\mathbb{B}}$ (Millipore) and eluted in $10 \mu \mathrm{l}$ 75:25:3 MeOH/water/ $\mathrm{HCOOH}(\mathrm{v} / \mathrm{v} / \mathrm{v})$ before mass spectrometry experiments.

\subsubsection{Mass spectrometric analyses}

MS and MS/MS experiments on tryptic peptides were performed on a quadrupole, orthogonal acceleration time-of-flight tandem mass spectrometer Q-TOF-PremierTM (Waters Corp., Milford, MA, USA). The proteins were ionized using nanoelectrospray ionization source heated at $80{ }^{\circ} \mathrm{C}$, in positive mode (ZSpray ${ }^{\mathrm{TM}}$ ). Samples were introduced in the mass spectrometer via nanoelectrospray glass capillaries (ProXeon) filled with $2-5 \mu \mathrm{L}$ of the protein or peptides solution and subsequently opened by breaking the tapered end of the tip under a microscope. The tension on the capillary was tuned manually between 2.1 and $2.6 \mathrm{kV}$ depending of the sample, and cone voltage set to $40 \mathrm{~V}$. MS experiments were performed in wide pass quadrupole mode, with the TOF data being collected between 200 and 2000 Th with a low collision energy of $5 \mathrm{eV}$. Argon was used as the collision gas. Scans were collected for $1 \mathrm{~s}$ and accumulated to increase the signal/noise ratio. MS/MS experiments were performed using a variable collision energy (20-32 eV), which was optimised for each precursor ion. Mass Lynx 4.1 was used both for acquisition and data processing. Deconvolution of multiply charged ions into neutral species was realized using MaxEnt1 in the mass range [ $40-50 \mathrm{kDa}$ ] with a resolution of 1.0 or $0.1 \mathrm{Da} / \mathrm{channel}$ for proteins and MaxEnt3 with an appropriate mass range for peptides. External calibration was performed in MS with clusters of phosphoric acid (0.01 M in 50:50 acetonitrile:water) immediately before each experimental set.

\subsection{Cloning and sequencing of EF-Tu Frateuria W-315}

On the basis of amino acid sequence identities between EF-Tu from Frateuria, from E. coli and from Ralstonia solanacearum, as 
determined by mass spectrometric analyses, an EF-Tu gene fragment of 445 bp was amplified using Pwo polymerase high fidelity PCR system (Roche Diagnostics) from Frateuria W-315 genomic DNA isolated following the instructions of E.Z.N.A bacterial DNA kit from OMEGA-Bio-tek and the $5^{\prime}$ primer GGTCACGTTCACCACGG and the $3^{\prime}$ primer CGTCGCCCGCGAAGTC.

This DNA fragment was sequenced by GenoScreen. The $5^{\prime}$ coding region of EF-Tu Frateuria was PCR amplified fom Frateuria W-315 genomic DNA using a $5^{\prime}$ primer sequence CCGCTGTCTGAA ATGTTCGGATAC of fusA1 gene, the upstream locus of tuf gene in genome sequence of Ralstonia solanacearum [17] and a 3'primer GATACCACGTGCCTTTTCTTCCGG corresponding to a $5^{\prime}$ region of the identified sequence of the PCR 445 bp fragment of Frateuria EF-Tu. The $3^{\prime}$ coding region of EF-Tu frateuria was PCR amplified fom Frateuria W-315 genomic DNA using a $5^{\prime}$ primer sequence GTGGACGACGCTGAACTGCTG corresponding to a $3^{\prime}$ region of the identified sequence of the PCR 445 bp fragment of Frateuria. EF-Tu and a $3^{\prime}$ primer GCCTTCAGGCGGATACGGAT corresponding to a sequence belonging to rpsJ gene downstream of the tuf gene in Ralstonia solanacearum genome sequence. The amplified fragments were purified and sequenced. The overlapped sequences allowed the determination of the complete ORF of the gene encoding EF-Tu from Frateuria W-315.

The coding sequence was amplified from Frateuria W-315 genomic DNA using the 5'primer CGTGGATCCGCAAAAGAGAAGTTCGAACGGACCAAG and the $3^{\prime}$ primer GATGAATTCTTAGTCGAGGATCTTGGCGACGACGCC respectively containing the BamH1 and EcoR1 sites (underlined) and cloned after digestion and purification into the BamH1-EcoR1 sites of the vector pGEX-2T. Accuracy of the amplification was controlled by sequencing of the cloned gene.

\subsection{Purification of recombinant Frateuria $W-315$ EF-Tu and E.coli EF-Tu mutants}

The different species of EF-Tu were overproduced in E. coli strain $\mathrm{DH} 5 \alpha$ as fusion with glutathione S-transferase [18]. The transformed $E$. coli strains were grown at $37^{\circ} \mathrm{C}$ in $2 \mathrm{~L}$ of LB rich medium containing $50 \mu \mathrm{g} \mathrm{ml}^{-1}$ ampicillin to $0.5 \mathrm{~A}_{600}$, after which induction with $0.1 \mathrm{mM}$ isopropyl-ß-D-thiogalacto-pyranoside took place at $23^{\circ} \mathrm{C}$ with incubation up to $17 \mathrm{~h}$. Under these conditions, high level of soluble EF-Tu was obtained.

After harvest, the cells were washed in PBS, sonicated 15 times for $10 \mathrm{~s}$ at $4{ }^{\circ} \mathrm{C}$ in $40 \mathrm{ml}$ buffer B $(50 \mathrm{mM}$ Tris- $\mathrm{HCl} \mathrm{pH} \mathrm{7.5,0.15} \mathrm{M}$ $\mathrm{NaCl}, 7 \mathrm{mM} \beta$-ME, $5 \mathrm{mM} \mathrm{MgCl} 2$ containing $0.5 \mathrm{mg} \mathrm{ml}^{-1}$ lysosyme, $100 \mu \mathrm{g} \mathrm{ml}^{-1}$ DNase, one tablet of complete mini EDTA free protease inhibitor cocktail (Roche Diagnostics), and centrifuged $(100,000 \mathrm{~g}$ for $30 \mathrm{~min}$ ). The extract supernatant was mixed for $30 \mathrm{~min}$ at $4{ }^{\circ} \mathrm{C}$ with $3 \mathrm{ml}$ glutathione sepharose 4 fast flow (GE healthcare). The suspension was washed several times with buffer $\mathrm{B}$, then with buffer C (25 mM Tris- $\mathrm{HCl} \mathrm{pH}$ 8.0, $2.5 \mathrm{mM} \mathrm{CaCl}_{2}, 1 \mathrm{mM} \mathrm{MgCl}_{2}, 30 \mathrm{mM}$ $\mathrm{KCl}, 10 \mu \mathrm{M}$ GDP) before incubation 3 times at $30^{\circ} \mathrm{C}$ for 15 min with $25 \mathrm{U}$ thrombin (Sigma) for removing $\mathrm{N}$-terminal fused glutathione S-transferase, $1 \mathrm{mM}$ Pefablock-SC (Roche Diagnostics) was then added. The last step of purification of the different EF-Tu species was a Mono Q-HR 5/5 chromatography (Äkta purifier system, GE Healthcare) with a linear gradient $50-300 \mathrm{mM} \mathrm{KCl}$ gradient $(50 \mathrm{ml}$ ) in buffer $\mathrm{D}\left(25 \mathrm{mM}\right.$ Tris- $\mathrm{HCl} \mathrm{pH} 7.5,1 \mathrm{mM} \mathrm{MgCl}_{2}, 7 \mathrm{mM} \beta$-ME, $10 \mu \mathrm{M}$ GDP). After concentration by ultrafiltration, EF-Tu preparations were dialysed and stored at $-25{ }^{\circ} \mathrm{C}$ in buffer D containing $50 \%$ glycerol.

\subsection{Site-directed mutagenesis}

Mutations were introduced with the QuickChange II XL Site-
Directed Mutagenesis Kit and by using the manufacturer (Stratagen) recommended protocol. As a substrate for the mutagenesis reactions we used pGtAM, a derivative of pGEX-2TtufA [19] which codes for a thrombin-cleavable glutathione $S$-transferase-fused E. coli EF-Tu. The mutagenic oligonucleotides for the amino acid substitutions were $5^{\prime}$-CCTACGGCGGTGAAGCTCGTGCATTCGACCAG$3^{\prime}$ for A42E, 5'-GCGGTGCTGCTCGTAAGTTCGACCAGATCG-3' for A45K, 5' -GCTGCTCGTGCATACGACCAGATCGATAACG-3' for F47Y, 5' CGTGCATTCGACGAGATCGATAACGCGCCG-3' for Q48E, 5'-GCTAAA ACCTACGGCGGTGAAGCTCGTAAGTTCGACCAGATCGATAACGC-3' for A42E/A45K; 5'-GGTGCTGCTCGTGCATACGACGAGATCGATAACGCG CCG-3' for F47Y/Q49E, 5'-GCTAAAACCTACGGCGGTGAAGCTCGTAA GTACGACGAGATCGATAACGC-3' for A42E/A45K/F46Y/Q48E (modified bases underlined) and their respective complementary mutagenic primers. Following transformation of competent XL10-Gold cells, minipreps on selected transformants were analyzed by sequencing to verify the presence of desired mutations and to check the absence of secondary mutations.

\subsection{Biological materials}

$\operatorname{Poly}(\mathrm{U})$ and tRNA ${ }^{\text {Phe }}$ from $E$. coli were from Sigma-Aldrich and L$\left.{ }^{14} \mathrm{C}(\mathrm{U})\right]$ Phenylalanine (18 GBq. $\left.\mathrm{mmol}^{-1}\right)$ ) from Perkin Elmer. Kirromycin was obtained from Gist-Brocades (Delft, The Netherlands), GE2270A from Drs E.Selva \& M. Denaro, (Lepetit Research Centre), and Enacyloxin IIa and IVa from Dr T. Watanabe, while Pulvomycin was isolated according to Smith et al. [20], tRNA ${ }^{\text {Phe }}$ was aminoacylated using $\left[{ }^{14} \mathrm{C}(\mathrm{U})\right]$ Phenylalanine with an excess amount of partially purified phenylalanyl-tRNA synthetase from E. coli as in Ref. [21]. E. coli 70S Ribosomes were prepared as reported in Ref. [21]. EF-Ts was purified as recombinant protein [22].

Fus gene encoding EF-G was amplified from total cellular DNA from E. Coli strain MRE600 using the $5^{\prime}$ primer CACCATGGCTCGTACAACACCCATC and the $3^{\prime}$ primer TTTACCACGGGCTTCAATTACGGC. The amplified fragment was cloned into pET101/D-TOPO vector using Champion pET directional TOPO expression kit from invitrogen. 6His-tagged EF-G was expressed in BL21 Star (DE3) E coli strain. The culture induced at a cell density of $0,5 \mathrm{~A}_{600}$ with $0.2 \mathrm{mM}$ isopropyl-ß-D-thiogalacto-pyranoside was collected after $6 \mathrm{~h}$ of growth at $28^{\circ} \mathrm{C}$ in LB medium containing ampicillin. After harvest, cells were sonicated 12 times for $10 \mathrm{~s}$ at $4{ }^{\circ} \mathrm{C}$ in buffer $\mathrm{A}$ (20 mM sodium phosphate $\mathrm{pH} 7.5,0.5 \mathrm{M} \mathrm{NaCl}, 20 \mathrm{mM}$ imidazole, $5 \mathrm{mM} \beta$-ME) containing $0.5 \mathrm{mg} \mathrm{ml}^{-1}$ lysosyme, $100 \mu \mathrm{g} \mathrm{ml}^{-1}$ DNase, one tablet of complete mini EDTA free protease inhibitor cocktail (Roche Diagnostics), and centrifuged (100,000 g for $30 \mathrm{~min}$ ). Supernatant was applied on $1 \mathrm{ml}$ His GraviTrap affinity column (GE Healthcare) equilibrated with buffer $A$, the column was washed with the same buffer before elution of the protein in buffer $A$ containing $0.5 \mathrm{M}$ imidazole. The eluted fraction containing EF-G was dialysed against buffer B (25 mM Tris- $\mathrm{HCl} \mathrm{pH} 7.5,1 \mathrm{mM}$ $\mathrm{MgCl}_{2}, 60 \mathrm{mM} \mathrm{KCl}, 7 \mathrm{mM} \beta-\mathrm{ME}$ ) and loaded on MonoQ HR 5/5 column (Äkta purifier system, GE Healthcare) using a linear $60-500 \mathrm{mM} \mathrm{KCl}$ gradient $(50 \mathrm{ml})$ in buffer B. After concentration by ultrafiltration, EF-G factor preparation was stored at $-20{ }^{\circ} \mathrm{C}$ in buffer B containing $50 \%$ glycerol. Pulvomycin, Kirromycin and enacyloxin were used as stable $20 \mathrm{mg} / \mathrm{ml}$ stock solution dissolved in methanol and kept at $-25^{\circ} \mathrm{C}$.

\subsection{Enzymatic assays}

Poly(Phe) synthesis was determined as incorporation of L$\left.{ }^{14} \mathrm{C}(\mathrm{U})\right]$ Phenylalanine into hot trichloroacetic acid-insoluble material as described in Ref. [23]. The reaction mixture contained $40 \mathrm{mM}$ Tris- $\mathrm{HCl} \mathrm{pH} 7.5,7 \mathrm{mM} \mathrm{MgCl}_{2}, 80 \mathrm{mM} \mathrm{NH} \mathrm{Nl}_{4}, 1 \mathrm{mM}$ dithiothreitol, $1 \mathrm{mM}$ ATP, $1 \mathrm{mM}$ phosphoenolpyruvate, $0.5 \mathrm{mM}$ GTP, 
$50 \mu \mathrm{g} \mathrm{ml}^{-1}$ pyruvate Kinase, $5 \mu \mathrm{M}$ tRNA ${ }^{\text {Phe }}$ (first charged during a 30 min incubation at $30{ }^{\circ} \mathrm{C}$ with a 2 fold excess of $\mathrm{L}-\left[{ }^{14} \mathrm{C}(\mathrm{U})\right]$ Phenylalanine ( $5 \mathrm{GBq} \cdot \mathrm{mmol}^{-1}$ ) and a saturating amount of partially purified phenylalanyl-tRNA synthetase), $3.5 \mu \mathrm{g} \operatorname{poly}(\mathrm{U})$ and antibiotic, $70 \mathrm{~S}$ ribosomes, elongation factors or S30 extract as described in legends to figures. After incubation aliquots were withdrawn spotted on glass fiber filters and hot trichoroacetic acid insoluble radioactivity was determined in a Wallac 1410 (Perkin Elmer life sciences) scintillation spectrometer.

Association rates of EF-Tu proteins with GDP or GTP and dissociation rates of EF-Tu•GDP and EF-Tu•GTP complexes were determined at $0{ }^{\circ} \mathrm{C}$ in standard buffer $50 \mathrm{mM}$ Tris $\mathrm{HCl} \mathrm{pH} \mathrm{7.5,5 \textrm {mM }}$ $\mathrm{MgCl}_{2}, 60 \mathrm{mM} \mathrm{NH}{ }_{4} \mathrm{Cl}, 0.05 \mathrm{mg} \cdot \mathrm{mL}^{-1} \mathrm{BSA}, 1 \mathrm{mM}$ dithiothreitol in absence or presence of $50 \mu \mathrm{M}$ antibiotic, using the nitrocellulose filtration procedure [24].

For the determination of association rates, nucleotide free EF-Tu proteins were prepared after incubation of pure EF-Tu•GDP proteins $(100-150 \mathrm{pmol}$ in $30 \mu \mathrm{l})$ for $20 \mathrm{~min}$ at $30^{\circ} \mathrm{C}$ in $25 \mathrm{mM}$ Tris$\mathrm{HCl}, \mathrm{pH} 7.5,100 \mathrm{mM}\left(\mathrm{NH}_{4}\right)_{2} \mathrm{SO}_{4}, 10 \mathrm{mM}$ NaEDTA, $1 \mathrm{mM}$ dithiothreitol. The solution was passed through a Sephadex G25 medium column $(18 \times 0.4 \mathrm{~cm})$ at $4{ }^{\circ} \mathrm{C}$ equilibrated with in $25 \mathrm{mM}$ Tris- $\mathrm{HCl}$, pH 7.5, $500 \mathrm{mM}\left(\mathrm{NH}_{4}\right)_{2} \mathrm{SO}_{4}, 0.5 \mathrm{mM}$ NaEDTA, $7 \mathrm{mM} \beta$-mercaptoethanol. $0.25 \mathrm{mg} \mathrm{mL}^{-1}$ BSA. A final concentration of $6 \mathrm{mM} \mathrm{MgCl}_{2}$ was added to the GDP-free EF-Tu containing fraction.

Prior to each GTP-containing assay, $5 \mu \mathrm{M}\left[{ }^{3} \mathrm{H}\right] \mathrm{GTP}$ (specific activity, $160 \mathrm{~Bq} \mathrm{pmol}{ }^{-1}$, Perkin Elmer life sciences) was preincubated in standard buffer for $10 \mathrm{~min}$ at $30^{\circ} \mathrm{C}$ with $10 \mu \mathrm{g}$ pyruvate kinase and $0.4 \mathrm{mM}$ phosphoenolpyruvate.

Association rates of EF-Tu with GTP or GDP were measured in standard buffer containing 2-30 nM nucleotide-free EF-Tu and 5-100 nM [ $\left.{ }^{3} \mathrm{H}\right]$ GTPor $4-10 \mathrm{nM}\left[{ }^{3} \mathrm{H}\right]$ GDP (specific activity, $200 \mathrm{~Bq}$ $\mathrm{pmol}^{-1}$, Perkin Elmer life sciences). The reaction was started with labeled nucleotide. Aliquots of the reaction mixture were withdrawn every $10 \mathrm{~s}$ for 2 -min period and filtered on nitrocellulose discs that were washed twice with $3 \mathrm{ml}$ ice-cold $25 \mathrm{mM}$ Tris- $\mathrm{HCl} \mathrm{pH}$ 7.5, $10 \mathrm{mM} \mathrm{MgCl} 2,60 \mathrm{mM} \mathrm{NH} 4 \mathrm{Cl} ; 7 \mathrm{mM} \beta$-mercaptoethanol. The nitrocellulose membranes were then counted for radioactivity. The apparent second order rate constants for association of EF-Tu products and guanine nucleotides were calculated according to the equation $1 /(b-a) \ln [\mathrm{a}(b-x) / b(a-x)]=k^{\prime}{ }_{+1} t$ were $a$ is the initial concentration of $\left[{ }^{3} \mathrm{H}\right] \mathrm{GTP}$ or $\left[{ }^{3} \mathrm{H}\right] \mathrm{GDP}, b$ is the initial concentration of nucleotide-free EF-Tu proteins and $x$ is the concentration of EFTu• $\left[{ }^{3} \mathrm{H}\right]$ GTP or EF-Tu• $\left[{ }^{3} \mathrm{H}\right]$ GDP complexes formed at the different time, $t$. The initial concentration $b$ was determined from the radioactivity bound to each GDP-free EF-Tu products in the presence of a saturating amount of $\left[{ }^{3} \mathrm{H}\right] \mathrm{GTP}$ or $\left[{ }^{3} \mathrm{H}\right]$ GDP after $10 \mathrm{~min}$ incubation in standard buffer at $30^{\circ} \mathrm{C}$.

For determination of dissociation rate constants $\left(k_{-1}\right)$, preformed labeled EF-Tu• $\left[{ }^{3} \mathrm{H}\right] \mathrm{GTP}$ or EF-Tu $\bullet\left[{ }^{3} \mathrm{H}\right] \mathrm{GDP}$ complexes were prepared by incubating for $15 \mathrm{~min}$ at $30^{\circ} \mathrm{C} 5 \mu \mathrm{M}$ EF-Tu•GDP in $25 \mathrm{mMTris}-\mathrm{HCl}$ $\mathrm{pH} 7.5,5 \mathrm{mM}$ EDTA, $1 \mathrm{mM}$ dithiothreitol and $0.05 \mathrm{mg} \mathrm{mL}^{-1}$ BSA with $10 \mu \mathrm{M}\left[{ }^{3} \mathrm{H}\right] \mathrm{GTP}$ or $15 \mu \mathrm{M}\left[{ }^{3} \mathrm{H}\right] \mathrm{GDP}\left(400 \mathrm{~Bq} \mathrm{pmol}^{-1}\right)$, then stabilized by adding $10 \mathrm{mM} \mathrm{MgCl} 2$ in standard buffer. The dissociation rates was started with $250 \mathrm{nM}$ preformed EF-Tu॰ $\left[{ }^{3} \mathrm{H}\right] \mathrm{GTP}$ or EF-Tu $\bullet\left[{ }^{3} \mathrm{H}\right]$ GDP complex in $95 \mu \mathrm{l}$ reaction mixture containing a 1000 fold excess of the corresponding nonlabeled nucleotide. At time intervals aliquots $(10 \mu \mathrm{l})$ were filtered on nitrocelluloses discs that were then washed and counted as described above.

For GTPase activity EF-Tu[ $\left.\gamma-{ }^{32} \mathrm{P}\right] \bullet \mathrm{GTP}$ complexes were preformed by incubating around $2 \mu \mathrm{M}$ EF-Tu॰GDP at $30^{\circ} \mathrm{C}$ for $10 \mathrm{~min}$ with $100 \mu \mathrm{M}\left[\gamma_{-}{ }^{32} \mathrm{P}\right] \bullet \mathrm{GTP}\left(20 \mathrm{~Bq}\right.$. pmol $\left.{ }^{-1}\right)$ in $25 \mathrm{mMTris}-\mathrm{HCl}$ ph 7.5 , $5 \mathrm{mM}$ EDTA, $1 \mathrm{mM}$ dithiothreitol and $0.05 \mathrm{mg} \mathrm{mL}^{-1} \mathrm{BSA}$ and then stabilized by the addition of $10 \mathrm{mM} \mathrm{MgCl} 2$.

GTPase activity was determined in $82 \mu \mathrm{l}$ reaction mixture containing $40 \mathrm{mM}$ Tris- $\mathrm{HCl} \mathrm{pH} 7.5,7 \mathrm{mM} \mathrm{MgCl}_{2}, 80 \mathrm{mM} \mathrm{NH}_{4} \mathrm{Cl}, 1 \mathrm{mM}$
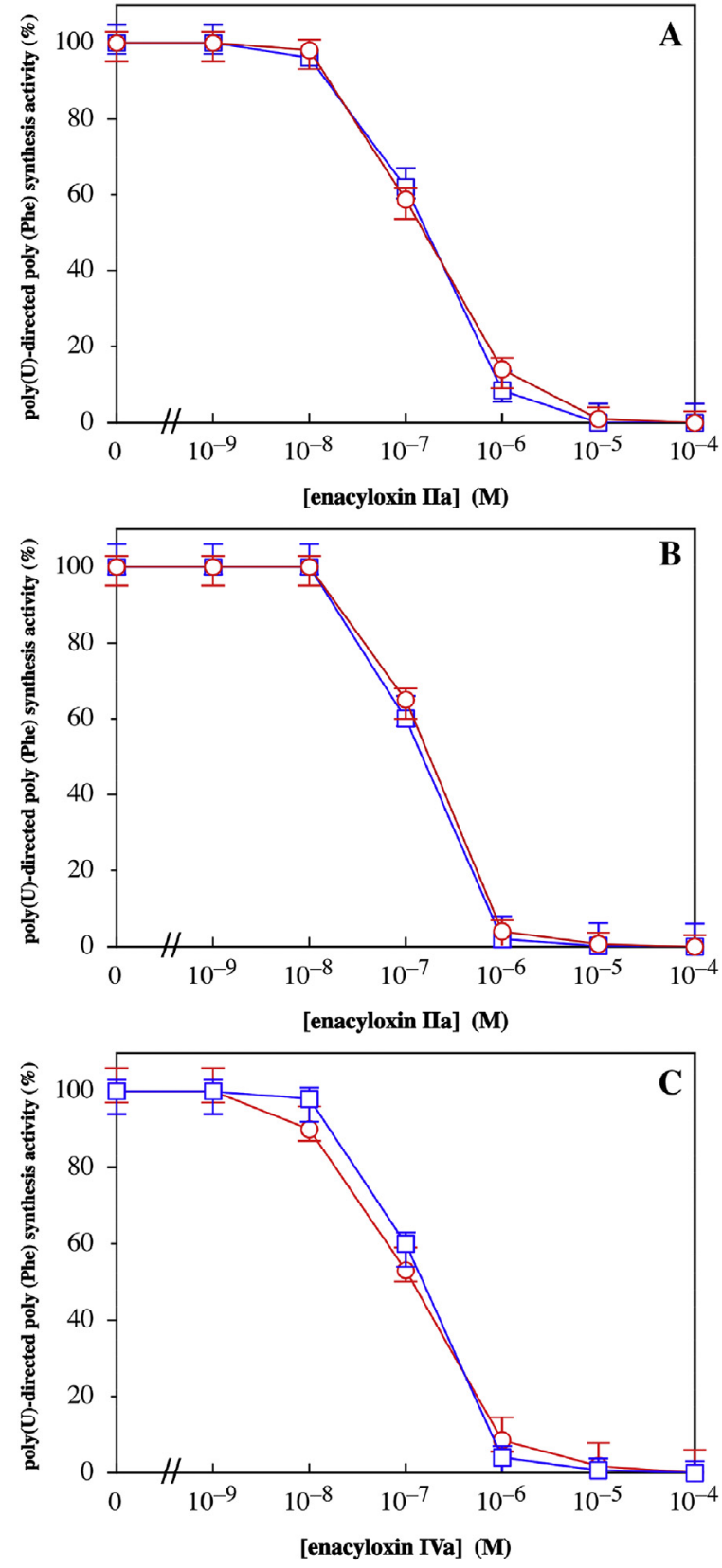

Fig. 1. Poly(U)-directed poly(phe) synthesis as function of increasing concentrations of enacyloxin determined $(A)$ in the presence of enacyloxin Ila and purified components of the translational machinery including purified EF-Tu from $E$. coli $(\bigcirc)$ or purified EFTu from Frateuria W315 ( $\sqsubset$ ); (B) in the presence of enacyloxin Ila and S30 extracts from E. coli $(\bigcirc)$ or from Frateuria W315 ( $\square)$, (C) in the presence of enacyloxin IVa and S30 extracts from E. coli ( $\$$ ) or from Frateuria W315 ( $\sqsubset$ ). The final reaction mixture $(70 \mu \mathrm{l})$ described in the Methods contained the indicated concentrations of the two forms of Enacyloxin and in A: $100 \mathrm{nM} \mathrm{EF-G,} 220 \mathrm{nM} 70 \mathrm{~S}$ ribosomes, $250 \mathrm{nM}$ EF-Tu from E. coli or from Frateuria W315, $250 \mathrm{nM}$ EF-Ts. In B and C: $80 \mu \mathrm{g}$ S30 extracts from E. Coli MRE600 or from Frateuria W315. The reaction was started by the addition of $\left[{ }^{14} \mathrm{C}\right] \mathrm{PhetRNA} \mathrm{Phe}^{\mathrm{Ph}}$ and stopped after 6 min incubation at $30{ }^{\circ} \mathrm{C}$ under conditions of linear Phe incorporation. The results are expressed as a percentage of the activity obtained in the absence of the antibiotic and are the average of three independent experiments.

dithiothreitol, 200-300 nM preformed EF-Tu[ $\left.\gamma-{ }^{32} \mathrm{P}\right] \bullet \mathrm{GTP}$ complex with or without $50 \mu \mathrm{M}$ antibiotic. The hydrolysis of $\left[\gamma-{ }^{32} \mathrm{P}\right] \bullet \mathrm{GTP}$ was measured at $30^{\circ} \mathrm{C}$ by following the liberation of $\gamma-{ }^{32} \mathrm{Pi}$ in time using the charcoal method: $10 \mu \mathrm{l}$ aliquots were withdrawn and the reaction was stopped with $300 \mu \mathrm{l}$ of a $4 \%$ suspension of activated charcoal in $20 \mathrm{mM} \mathrm{H}_{3} \mathrm{PO}_{4}$.

After centrifugation the radioactivity in $200 \mu$ l supernatant was 
counted. The amount of GTP hydrolysis obtained in the absence of EF-Tu was subtracted.

\section{Results}

3.1. Effect of enacyloxin on protein biosynthesis in vitro sustained by EF-Tu from Frateuria $W-315$

Elongation factor Tu was previously shown to be the specific target of enacyloxin [9]. The antibiotic hinders the release of EFTu•GDP from the ribosome after GTP hydrolysis, thus inhibiting its recycling and peptide bound formation [9].

After having purified to homogeneity EF-Tu from Frateuria W315 following the procedure described in Methods, we have determined the effect of enacyloxin by using an in vitro poly(Phe) synthesis assay catalysed by purified translational components from E. coli.

We expected that the EF-Tu-dependent poly(Phe) synthesis activity of Frateuria sp. W-315 would be insensitive to increasing concentrations of enacyloxin Ila, insofar as this antibiotic is produced by the strain itself. Unexpectedly, poly(U)-dependent poly(Phe) synthesis assay with EF-Tu from Frateuria sp. W-315 was inhibited by the antibiotic similarly to the one with $E$. coli EF-Tu at a concentration of about $0.2 \mu \mathrm{M}\left(\mathrm{IC}_{50}\right)$ (Fig 1A).

The effect of enacyloxin Ila was then measured on poly(Phe) synthesis sustained by S30 extract from E. coli and Frateuria W-315, respectively. As shown in (Fig 1B), the inhibitory effect of enacyloxin Ila was almost the same as that obtained in the purified system.

In the early phase of production of enacyloxins by Frateuria W315 , enacyloxin IVa was first secreted, followed by its conversion to enacyloxin IIa [25] by an extracellular quinoprotein oxidase. The possibility that Frateuria EF-Tu would be more resistant to the intermediate Enacyloxin IVa than to the end product Enacyloxin IIa was checked by measuring the effect of Enacyloxin IVa on the
poly(U)-dependent poly(Phe) synthesis assay sustained by S30 extract from Frateuria sp. W-315. As shown in Fig. 1C, the sensitivity was the same that with the final product. These results strongly suggest that antibiotic resistance of enacyloxins producing Frateuria W-315 is not due neither to EF-Tu nor to other components from the translation apparatus like the ribosomes, but rather to other independent factors.

\subsection{Sequencing, cloning and purification of cloned EF-Tu from Frateuria $W-315$}

Purified EF-Tu from Frateuria W-315 was subjected to trypsin digestion and mass spectrometric analyses (Fig. 2).

First, primary structure similarities between EF-Tu Frateuria W315 and EF-Tu Ralstonia solanacearum, an aerobic non sporing gram negative plant pathogenic bacterium [17] were identified with the FASTA program. Thereafter, EF-Tu gene was PCR amplified from Frateuria W-315 genomic DNA following the procedure described in Methods, and sequenced. The DNA sequence (Fig. 3) shows an ORF of 1191 nucleotides corresponding to 396 amino acids. This protein is larger than the product of tufA from E. coli by only two residues.

Alignment of the amino acid sequence of EF-Tu from E. coli with those of Frateuria and Ralstonia solanacearum indicates on average $80 \%$ identical amino acid residues and 9.7\% conservative replacements between EF-Tu Frateuria and EF-Tu E. coli, on one hand, and $97 \%$ identity and $1.7 \%$ conservative replacement between EF-Tu Frateuria and EF-Tu Ralstonia solanacearum, on the other hand (Fig. 4). These strong primary structure similarities between EF-Tu from different origins are consistent with the fact that this factor is essential for the translation process in all kingdoms of life. The major difference in the primary structure between the three aligned factors resides in fragment 36-48 located in the so called "effector region".

The coding sequence amplified from Frateuria genomic DNA was

\begin{tabular}{|c|c|c|c|c|}
\hline fragment & Mr expt & Mr theor & delta & sequence \\
\hline $9-25$ & 1794.9512 & 1794.9489 & 0.0023 & R.TKPHVNVGTIGHVDHGK.T \\
\hline $26-39$ & 1375.7758 & 1375.7922 & -0.0164 & K.TTLTAAIATVLSSK.F \\
\hline $46-57$ & 1406.6519 & 1406.6565 & -0.0046 & K.KYDEIDAAPEEK.A \\
\hline $60-75$ & 1801.8802 & 1801.8958 & -0.0156 & R.GITINTAHIEYETANR.H \\
\hline $76-90$ & 1767.7575 & 1767.7787 & -0.0212 & R.HYAHVDCPGHADYVK.N Carbamidomethyl (C) \\
\hline $91-117$ & 2775.2483 & 2775.2812 & -0.0329 & K.NMITGAAQMDGAILVCSAADGPMPQTR.E Carbamidomethyl (C) \\
\hline $118-124$ & 850.4959 & 850.5025 & -0.0065 & R.EHILLAR.Q \\
\hline $138-155$ & 2164.9444 & 2164.969 & -0.0246 & K.CDMVDDAELLELVEMEVR.E Carbamidomethyl (C) \\
\hline $161-172$ & 1379.6541 & 1379.6609 & -0.0068 & K.YDFPGDDTPIIK.G \\
\hline $208-226$ & 2038.9889 & 2039.0034 & -0.0145 & R.AVDGTFLMPVEDVFSISGR.G \\
\hline $241-251$ & 1184.6574 & 1184.6652 & -0.0078 & K.VGEEIEIVGIK.A \\
\hline $256-265$ & 1200.5214 & 1200.5267 & -0.0053 & K.TTCTGVEMFR.K Carbamidomethyl (C) \\
\hline $266-282$ & 1809.0044 & 1809.0108 & -0.0064 & R.KLLDQGQAGDNVGILLR.G \\
\hline $267-282$ & 1680.8997 & 1680.9159 & -0.0162 & K.LLDQGQAGDNVGILLR.G \\
\hline $322-336$ & 2032.9453 & 2032.9697 & -0.0243 & R.HTPFFNNYRPQFYFR.T \\
\hline $337-348$ & 1259.6514 & 1259.6609 & -0.0095 & R.TTDVTGSIELPK.D \\
\hline $337-350$ & 1502.7725 & 1502.7828 & -0.0103 & R.TTDVTGSIELPKDK.E \\
\hline $349-364$ & 1761.857 & 1761.8641 & -0.0071 & K.DKEMVMPGDNVSITVK.L \\
\hline $349-364$ & 1777.8168 & 1777.859 & -0.0422 & K.DKEMVMPGDNVSITVK.L Oxidation (M) \\
\hline $351-364$ & 1518.7401 & 1518.7422 & -0.0021 & K.EMVMPGDNVSITVK.L \\
\hline $351-364$ & 1550.7246 & 1550.732 & -0.0074 & K.EMVMPGDNVSITVK.L 2 Oxidation (M) \\
\hline $365-376$ & 1311.7102 & 1311.722 & -0.0118 & K.LIAPIAMEEGLR.F \\
\hline $385-393$ & 800.4761 & 800.4756 & -0.0005 & R.TVGAGVVAK.I \\
\hline
\end{tabular}

Fig. 2. Mass spectrometric analyses of tryptic peptides from EF-Tu Frateuria W 315. 


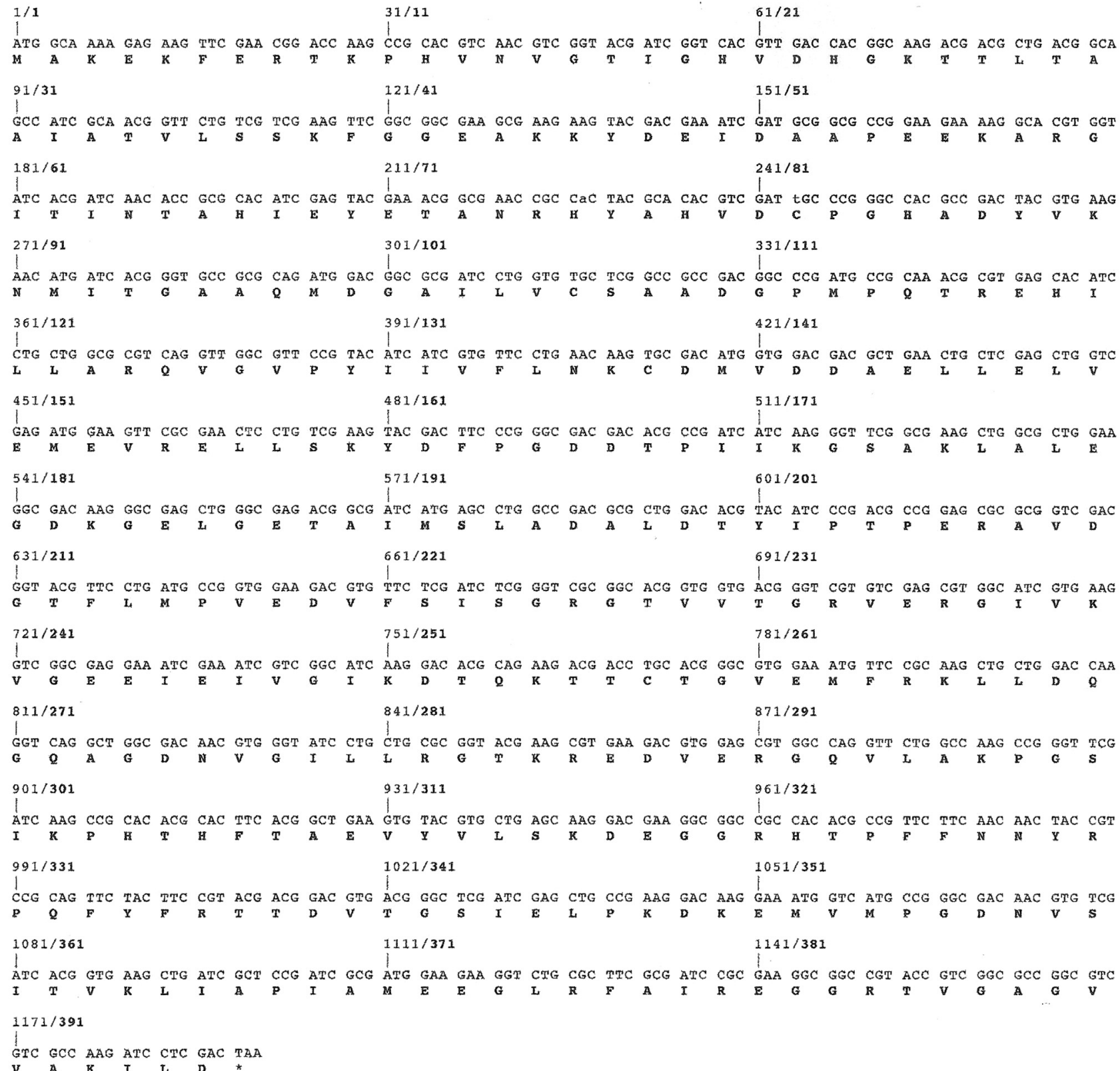

Fig. 3. Nucleotide sequence of the gene encoding EF-Tu from Frateuria W 315 and the amino acid sequence deduced.

cloned in pGEX-2T, overproduced as fusion with glutathione $S$ transferase in E. coli strain DH5 $\alpha$ and purified free of fused GST after thrombin treatment.

\subsection{Comparison of the dynamics of interaction between GDP or GTP and EF-Tu Frateuria or EF-Tu E.coli and the effects of antibiotics}

Table 1 shows the apparent association and dissociation rates constants and the derived dissociation constant $\left(K_{\mathrm{D}}^{\prime}=k^{\prime}{ }_{-1} / k^{\prime}{ }_{+1}\right)$ of EF-Tu•GDP Frateuria and EF-Tu•GDP E. coli complexes and the respective effect of the antibiotics enacyloxin, kirromycin, pulvomycin and GE2270A. These antibiotics are well known to act specifically on EF-Tu $[9,12,13,21,24,26]$. Due to a decrease of the dissociation rate and a modest increase of the association rate, the affinity of EF-Tu Frateuria for GDP was found to be 3 times higher than that of EF-Tu E. coli (Table 1).

Comparison of the effects of antibiotics on EF-Tu Frateuria and
EF-Tu E. coli revealed that enacyloxin, kirromycin and pulvomycin exert a stronger stimulation of the GDP dissociation rate on EF-Tu Frateuria, while the effects of the antibiotics on the GDP association rate were comparable for the two EF-Tu species. No effect on the dissociation rate was observed with GE2270A while it inhibited almost 4 fold the association rate for EF-Tu Frateuria. As for the interaction with GTP (Table 2), due to an increase of the association rate, the affinity of EF-Tu Frateuria was found to be 2.5 times higher than that for EF-Tu E. coli. All the used antibiotics were shown to increase the affinity of the two EF-Tu species for GTP. While each antibiotic was shown to reduce the GTP dissociation rates to approximately the same extent for the two EF-Tu species, their effects on the stimulation of the GTP association rate were lower for EF-Tu Frateuria than for EF-Tu E. coli. In particular, enacyloxin was shown to exert a stimulation more than 8 times lower. 


$$
\begin{aligned}
& \text { EF_Tu-Frat } \\
& \text { EF_Tu-Rals.so } \\
& \text { EF_Tu-EColi } \\
& \text { EF_Tu-Frat } \\
& \text { EF_Tu-Rals.so } \\
& \text { EF_Tu-EColi }
\end{aligned}
$$

EF_Tu-Frat EF_Tu-Rals.so EF_Tu-EColi

$$
\begin{aligned}
& \text { EF_Tu-Frat } \\
& \text { EF_Tu-Rals.so } \\
& \text { EF_Tu-EColi }
\end{aligned}
$$

$$
\begin{aligned}
& \text { EF_Tu-Frat } \\
& \text { EF_Tu-Rals.so } \\
& \text { EF_Tu-EColi }
\end{aligned}
$$

$$
\begin{aligned}
& \text { EF_Tu-Frat } \\
& \text { EF_Tu-Rals.so } \\
& \text { EF_Tu-EColi }
\end{aligned}
$$

$$
\begin{aligned}
& \text { EF_Tu-Frat } \\
& \text { EF_Tu-Rals.so } \\
& \text { EF_Tu-EColi }
\end{aligned}
$$

MAKEKFERTKPHVNVGTIGHVDHGKTTLTAAIATVLSSKFGGEAKKYDEIDAAPEEKARG 60 MAKEKFERTKPHVNVGTIGHVDHGKTTLTAAIATVLSSKFGGEAKKYDEIDAAPEEKARG 60 -SKEKFERTKPHVNVGTIGHVDHGKTTLTAAITTVLAKTYGGAARAFDQIDNAPEEKARG 59

$: * * * * * * * * * * * * * * * * * * * * * * * * * * * * * *: * * *: .:: * * \quad *:: * * * * \quad * * * * * * * *$

ITINTAHIEYETANRHYAHVDCPGHADYVKNMITGAAQMDGAILVCSAADGPMPQTREHI 120 ITINTAHIEYETANRHYAHVDCPGHADYVKNMITGAAQMDGAILVCSAADGPMPQTREHI 120 ITINTSHVEYDTPTRHYAHVDCPGHADYVKNMITGAAQMDGAILVVAATDGPMPQTREHI 119 $* * * * *: *: * *: * \ldots * * * * * * * * * * * * * * * * * * * * * * * * * * * * * * *:: *: * * * * * * * * * * *$

LLARQVGVPYI IVFLNKCDMVDDAELLELVEMEVRELLSKYDFPGDDTPI IKGSAKLALE 180 LLARQVGVPYIIVFLNKCDMVDDAELLELVEMEVRELLSKYDFPGDDTPIIKGSAKLALE 180 LLGRQVGVPYIIVFLNKCDMVDDEELLELVEMEVRELLSQYDFPGDDTPIVRGSALKALE 179 ** .******************** $* * * * * * * * * * * * * * *: * * * * * * * * * *:: * * * \quad * * *$

GDKGELGETAIMSLADALDTYIPTPERAVDGTFLMPVEDVFSISGRGTVVTGRVERGIVK 240 GDKGELGEVAIMNLADALDSYIPTPERAVDGTFLMPVEDVFSISGRGTVVTGRIERGIIK 240 GD--AEWEAKILELAGFLDSYIPEPERAIDKPFLLPIEDVFSISGRGTVVTGRVERGIIK 237 ** $\quad * * z_{0 *} * * *: * * * * * * *: . * *: *: * * * * * * * * * * * * * * *: * * * *: *$

VGEEIEIVGIKDTQKTTCTGVEMFRKLLDQGQAGDNVGILLRGTKREDVERGQVLAKPGS 300 VGEEIEIVGIKATQKTTCTGVEMFRKLLDQGQAGDNVGILLRGTKREDVERGQVLCKPGS 300 VGEEVEIVGIKETQKSTCTGVEMFRKLLDEGRAGENVGVLLRGIKREEIERGQVLAKPGT 297 $* * * *: * * * * * * * * *: * * * * * * * * * * * * *: *: * *: * * *: * * * * * * *:: * * * * * * . * * *:$

IKPHTHFTAEVYVLSKDEGGRHTPFFNNYRPQFYFRTTDVTGSIELPKDKEMVMPGDNVS 360 IKPHTHFTGEVYILSKDEGGRHTPFFNNYRPQFYFRTTDVTGSIELPKDKEMVMPGDNVS 360 IKPHTKFESEVYILSKDEGGRHTPFFKGYRPQFYFRTTDVTGTIELPEGVEMVMPGDNIK 357 $* * * * *: * \quad . * * *: * * * * * * * * * * * * *: . * * * * * * * * * * * * * *: * * * *: . * * * * * * * *: 。$

ITVKLIAPIAMEEGLRFAIREGGRTVGAGVVAKILD 396 ITVKLIAPIAMEEGLRFAIREGGRTVGAGVVAKIIE 396 MVVTLIHPIAMDDGLRFAIREGGRTVGAGVVAKVLG 393 $:$. $. * * * * * *:: * * * * * * * * * * * * * * * * * * * *::$

Fig. 4. Multiple sequence alignment of EF-Tu Frateuria W 315, EF-Tu Ralstonia solanacearum and EF-Tu E. coli.
3.4. Stimulation of poly(Phe) synthesis by EF-Tu E. coli or EF-Tu

\begin{tabular}{|c|c|c|c|c|}
\hline & $\begin{array}{l}\text { Dissociation rate } \\
\text { constant }\left(k_{-1}^{\prime}\right) 10^{-4} \mathrm{~s}^{-1}\end{array}$ & $\begin{array}{l}\text { Dissociation } \\
\text { half-lives (min) }\end{array}$ & $\begin{array}{l}\text { Association rate } \\
\text { constant }\left(k_{+1}^{\prime}\right) 10^{5} \mathrm{M}^{-1} \mathrm{~s}^{-1}\end{array}$ & $\begin{array}{l}\text { Dissociation } \\
\text { constant }\left(K_{D}^{\prime}\right)(\mathrm{nM})\end{array}$ \\
\hline EF-Tu•GDP $($ E.coli $)$ & $4.9 \pm 0.04$ & 22 & $2.5 \pm 0.02$ & 2 \\
\hline EF-Tu•GDP (Frateuria W-315) & $2.0 \pm 0.03$ & 57 & $3.0 \pm 0.03$ & 0.7 \\
\hline EF-Tu•GDP $($ E.coli $)+$ enacyloxin & $7.2 \pm 0.2$ & 16 & $4.0 \pm 0.12$ & 1.8 \\
\hline EF-Tu•GDP Frateuria W-315) + enacyloxin & $19.6 \pm 1.2$ & 6 & $4.3 \pm 0.16$ & 4.5 \\
\hline EF-Tu•GDP $($ E.coli $)+$ kirromycin & $7.7 \pm 0.3$ & 14 & $5.5 \pm 0.3$ & 1.4 \\
\hline EF-Tu•GDP (Frateuria W-315) + kirromycin & $16.6 \pm 0.9$ & 6.7 & $4.7 \pm 0.15$ & 3.6 \\
\hline EF-Tu•GDP $($ E.coli $)+$ pulvomycin & $14 \pm 0.4$ & 6.5 & $6.1 \pm 0.2$ & 2.3 \\
\hline EF-Tu•GDP (Frateuria $\mathrm{W}-315)+$ pulvomycin & $32.6 \pm 2.1$ & 3.5 & $11 \pm 0.9$ & 3 \\
\hline EF-Tu•GDP $($ E.coli $)+$ GE2270A & $5.2 \pm 0.12$ & 21 & $1.8 \pm 0.05$ & 2.9 \\
\hline EF-Tu•GDP (Frateuria W-315) + GE2270A & $1.9 \pm 0.03$ & 58 & $0.8 \pm 0.02$ & 2.4 \\
\hline
\end{tabular}
Frateuria: effects of mutations in the "effector region »

The main difference in amino acid sequence between EF-Tu E. coli and EF-Tu Frateuria is located in the region 36-48 which is part of the so-called « effector region ».

We have constructed different mutants of EF-Tu E. coli with the help of site directed mutagenesis by changing one or several residues of EF-Tu E. coli by the corresponding residues of EF-Tu Frateuria.

Poly(Phe) synthesis determined as a function of increasing concentrations of EF-Tu E. coli or EF-Tu Frateuria shows that the former is 6 times more efficient in the stimulation of $\operatorname{Poly}(U)$ directed poly(Phe) synthesis (Fig. 5).

Taking the extent of activation as a measure of productive interaction between EF-Tu and the programmed-ribosome we

Table 1

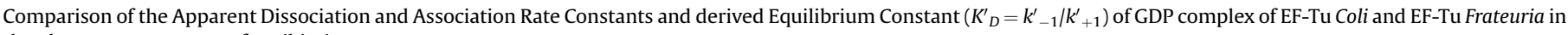
the absence or presence of antibiotics. 
Table 2

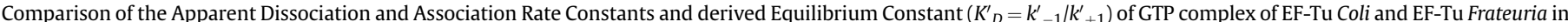
the absence or presence of antibiotics.

\begin{tabular}{|c|c|c|c|c|}
\hline & $\begin{array}{l}\text { Dissociation rate } \\
\text { constant }\left(k_{-1}^{\prime}\right) 10^{-4} \mathrm{~s}^{-1}\end{array}$ & $\begin{array}{l}\text { Dissociation } \\
\text { half-lives (min) }\end{array}$ & $\begin{array}{l}\text { Association rate } \\
\text { constant }\left(k_{+1}^{\prime}\right) 10^{4} \mathrm{M}^{-1} \mathrm{~s}^{-1}\end{array}$ & $\begin{array}{l}\text { Dissociation } \\
\text { constant }\left(K_{D}^{\prime}\right)(\mathrm{nM})\end{array}$ \\
\hline EF-Tu•GTP $($ E.coli $)$ & $18 \pm 0.9$ & 6.3 & $0.76 \pm 0.01$ & 237 \\
\hline EF-Tu•GTP (Frateuria W-315) & $21 \pm 1.7$ & 5.3 & $2.2 \pm 0.03$ & 95 \\
\hline EF-Tu•GTP $($ E.coli $)+$ enacyloxin & $5.5 \pm 0.09$ & 20.5 & $2.3 \pm 1.6$ & 2.5 \\
\hline EF-Tu•GTP (Frateuria W-315)+ enacyloxin & $6.3 \pm 0.18$ & 17.5 & $7.8 \pm 0.2$ & 8 \\
\hline EF-Tu•GTP $($ E.coli $)+$ kirromycin & $1.3 \pm 0.02$ & 89 & $15.2 \pm 0.9$ & 0.86 \\
\hline EF-Tu•GTP (Frateuria W-315) + kirromycin & $1.0 \pm 0.02$ & 109 & $21 \pm 1.4$ & 0.48 \\
\hline EF-Tu•GTP $($ E.coli $)+$ pulvomycin & $1.7 \pm 0.03$ & 68.5 & $47 \pm 2.6$ & 0.36 \\
\hline EF-Tu•GTP (Frateuria W-315) + pulvomycin & $1.7 \pm 0.04$ & 67 & $75.5 \pm 5.4$ & 0.22 \\
\hline EF-Tu•GTP $($ E.coli $)+$ GE2270A & $1.0 \pm 0.01$ & 107 & $5.2 \pm 0.4$ & 1.9 \\
\hline EF-Tu•GTP (Frateuria W-315) + GE2270A & $0.93 \pm 0.02$ & 124 & $4.9 \pm 0.3$ & 2 \\
\hline
\end{tabular}

determined by double reciprocal plots the concentration inducing half maximum activation $\left(K_{\mathrm{a}}\right)$ and the corresponding $\mathrm{V}_{\max }$.

The concentration inducing half maximal activation were calculated to be $12 \mathrm{nM}$ for EF-Tu E. coli and $71 \mathrm{nM}$ for EF-Tu Frateuria. The maximal efficiency $\left(\mathrm{V}_{\max }\right)$ was the same for both EF-Tu species. (Fig. 5 and Table 3).

We analyzed the effect of various amino acid changes in EF-Tu $E$. coli, by replacing one or several amino acid residues in the 42-46 fragment by those of EF-Tu Frateuria.

As shown in Table 3 and Fig. 5, none of these substitutions affect the maximal level of activation. The single substitutions F46Y or Q48E do not modify the $K_{\mathrm{a}}$ value while the individual mutations A42E and A45K reduced slightly the affinity for the programmed ribosome. The effect of the double substitutions A42E/A45K andF46Y/Q48E is more pronounced in increasing the $K_{\mathrm{a}}$ value.

Introduction of the four mutations A42E/A45K/F46Y/Q48E increased the $K_{\mathrm{a}}$ to a value comparable with that of EF-Tu Frateuria.

\subsection{Intrinsic GTPase activity: effect of Kirromycin, Enacyloxin, aminoacyl-tRNA and ribosome}

GTPase activity was determined with preformed EF-Tu[ $\left.\gamma-{ }^{32} \mathrm{P}\right] \bullet$

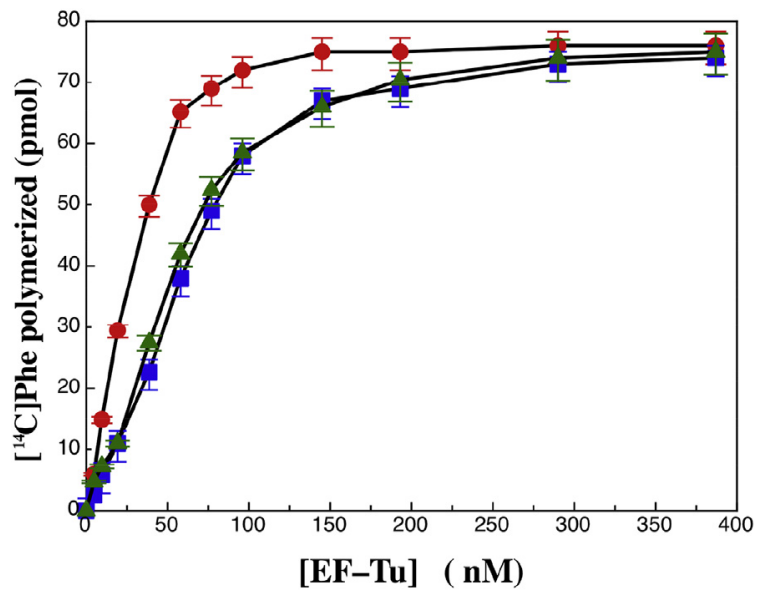

Fig. 5. Comparison of Poly(U)-directed poly(phe) activation dependent of increasing

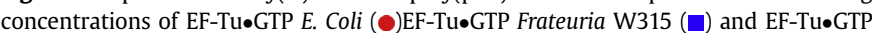
Coli A42E/A45K/F46Y/Q48E (A). The final reaction mixture $(30 \mu \mathrm{l})$ described in the Methods contained the indicated concentrations of the different species of EF-Tu in their GTP complex form, $0.25 \mu \mathrm{M}$ EF-G, $0.2 \mu \mathrm{M}$ EF-Ts, $0.3 \mu \mathrm{M}$ 70S ribosome. EF-Tu•GTP complexes were preformed by incubating $2 \mu \mathrm{M}$ EF-Tu॰GDP at $30{ }^{\circ} \mathrm{C}$ for $10 \mathrm{~min}$ with $0.5 \mathrm{mM}$ GTP, $1 \mathrm{mM} 1 \mathrm{mM}$ Phosphoenolpyruvate, $50 \mu \mathrm{g} \mathrm{ml}^{-1}$ pyruvate Kinase in $40 \mathrm{mM}$ Tris- $\mathrm{HCl} \mathrm{pH} 7.5,7 \mathrm{mM} \mathrm{MgCl} 2,80 \mathrm{mM} \mathrm{NH}_{4} \mathrm{Cl}, 1 \mathrm{mM}$ dithiothreitol. The reaction was started by the addition of $\left[{ }^{14} \mathrm{C}\right] \mathrm{PhetRNA}{ }^{\text {Phe }}$ and stopped after $10 \mathrm{~min}$ incubation at $30{ }^{\circ} \mathrm{C}$ under conditions of linear phe incorporation.
GTP complex and a large excess of $\left[\gamma^{-}{ }^{32} \mathrm{P}\right] \bullet \mathrm{GTP}$.

GTPase activities of EF-Tu E. coli and EF-Tu Frateuria were compared in the presence or in the absence of physiological partners like aa-tRNA or ribosomes, as well as in the presence of the antibiotics Kirromycin and Enacyloxin.

As shown in Table 4 and Fig. 6, intrinsic GTPase activity of EF-Tu Frateuria is almost 7 times higher than that of EF-Tu E. coli.

In our conditions, kirromycin enhances 10 times the $k_{\text {cat }}$ value of the intrinsic GTPase activity of EF-Tu E. coli, while it enhances only 5 times the one of EF-Tu Frateuria. In contrast the antibiotic enacyloxin modestly enhances the GTPase activity of EF-Tu E. coli by a factor 2 and that of EF-Tu Frateuria by a factor of 3.5.

In the presence of phenylalanyl-tRNA ${ }^{\text {Phe }}$, the intrinsic GTPase activities of EF-Tu E. coli and EF-Tu Frateuria were reduced by 50\% and $30 \%$, respectively, in agreement with previously reported data $[27,28]$.

In the presence of kirromycin, the ribosomes stimulate the GTPase activity of the two EF-Tu species by a factor 8 (Table 4). However, in the presence of enacyloxin, the GTPase activities of EFTu E. coli and EF-Tu Frateuria were stimulated by the ribosomes by factors 40 and 13 , respectively.

Among the naturally occurring amino acid changes between EFTu E. coli and EF-Tu Frateuria in the "effector region", we have focused on the following: Ala-42, Ala-45, Phe-46 and Gln-48 in EF$\mathrm{Tu} E$. coli correspond respectively to Glu- 42 , Lys- 45 , Tyr- 46 and Glu48 in EF-Tu Frateuria. At each of these four positions, we have replaced the amino acid residue present in the E. coli factor by the one present in the Frateuria factor, and we have analyzed the effects of the mutations on the intrinsic GTPase activity of these mutant E. coli EF-Tu species.

The single A45K substitution did not modify the intrinsic GTPase activity of EF-Tu E. coli. In contrast, a 2-3 fold stimulation was

Table 3

Determination of the concentration of EF-Tu inducing half maximum activation $\left(K_{\mathrm{a}}\right)$ in Poly(U)-directed poly(phe) synthesis and corresponding $\mathrm{V}_{\max }$. Comparison between EF-Tu Coli, EF-Tu Frateuria, and the various mutants of EF-Tu Coli in the effector region ». The assays were performed as described in legend to Fig. 5. Values were calculated from double-reciprocal plots.

\begin{tabular}{lrc}
\hline EF-Tu•GTP species & \multicolumn{1}{c}{$K_{\mathrm{a}}(\mathrm{nM})$} & $\mathrm{V}_{\max }\left(\mathrm{pmol}^{-1} \mathrm{~min}^{-1}\right)$ \\
\hline EF-Tu E.coli & $12 \pm 0.8$ & $8 \pm 0.6$ \\
EF-Tu Frateuria W-315 & $71 \pm 4.2$ & $8.4 \pm 0.5$ \\
EF-Tu E42 & $48 \pm 3.2$ & $8.3 \pm 0.5$ \\
EF-Tu K45 & $37 \pm 2.6$ & $8.2 \pm 0.6$ \\
EF-Tu E42/K45 & $54 \pm 4.8$ & $8.4 \pm 0.7$ \\
EF-Tu Y46 & $13.5 \pm 1.6$ & $8.0 \pm 0.9$ \\
EF-Tu E48 & $18 \pm 2.1$ & $8.2 \pm 0.9$ \\
EF-Tu Y46/E48 & $63 \pm 4.8$ & $8.2 \pm 0.6$ \\
EF-Tu E42/K45/Y46/E48 & $67 \pm 5.1$ & $8.6 \pm 0.7$ \\
\hline
\end{tabular}


Table 4

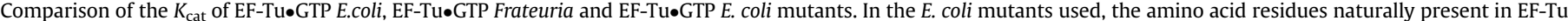

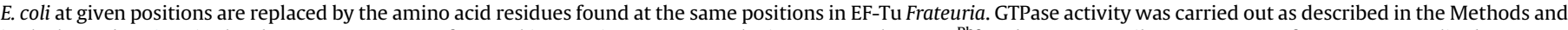

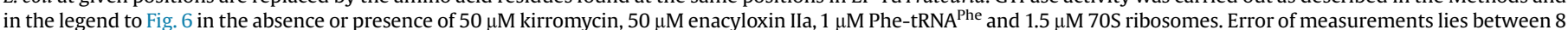
and $15 \%$.

\begin{tabular}{|c|c|c|c|c|c|c|}
\hline & $\begin{array}{l}\text { Intrinsic } \\
k_{\text {cat }} \times 10^{2}\left(\mathrm{~min}^{-1}\right)\end{array}$ & $\begin{array}{l}\text { plus kirromycin } \\
k_{\text {cat }} \times 10^{2}\left(\mathrm{~min}^{-1}\right)\end{array}$ & $\begin{array}{l}\text { plus enacyloxin } \\
k_{\text {cat }} \times 10^{2}\left(\mathrm{~min}^{-1}\right)\end{array}$ & $\begin{array}{l}\text { plus PhetRNAPhe } \\
k_{\text {cat }} \times 10^{2}\left(\mathrm{~min}^{-1}\right)\end{array}$ & $\begin{array}{l}\text { plus ribosome plus } \\
\text { kirromycin } k_{\text {cat }} \times 10^{2}\left(\mathrm{~min}^{-1}\right)\end{array}$ & $\begin{array}{l}\text { plus ribosome plus } \\
\text { enacyloxin } k_{\text {cat }} \times 10^{2}\left(\mathrm{~min}^{-1}\right)\end{array}$ \\
\hline EF-Tu E. coli & 1.4 & 13.5 & 2.5 & 0.71 & 118 & 103 \\
\hline EF-Tu Frateuria W-315 & 9.4 & 44.5 & 32 & 6.6 & 346 & 420 \\
\hline EF-Tu E. coli A42E & 3.0 & 50 & 21.2 & & & \\
\hline EF-Tu E. coli A45K & 1.6 & 20 & 4 & & & \\
\hline EF-Tu E. coli A42E/A45K & 1.6 & 27 & 8 & & & \\
\hline EF-Tu E. coli F46Y & 4.3 & 63 & 11 & & & \\
\hline EF-Tu E. coli $\mathrm{Q} 48 \mathrm{E}$ & 3.0 & 40 & 13.6 & & & \\
\hline EF-Tu E. coli F46Y/Q48E & 4.9 & 96 & 26.4 & & & \\
\hline $\begin{array}{l}\text { EF-Tu E. coli A42E/ } \\
\text { A45K/F46Y/Q48E }\end{array}$ & 9.5 & 160 & 62 & & & \\
\hline
\end{tabular}

observed with the single A42E, F46Y, Q48E and the double F46Y/ Q48E substitutions. Finally, up to a 7 fold stimulation was observed with the quadruple substitution (mutant A42E/A45K/F46Y/Q48E).

Stimulation of the intrinsic GTPase activity of EF-Tu E. coli in the presence of kirromycin was increased 3.7, 3 and 5 fold respectively, by the single A42E, Q48E and F46Y substitutions, while the stimulation was increased 7 and 12 fold respectively, by the double (F46Y/Q48E) and the quadruple (A42E/A45K/F46Y/Q48E) substitutions.

While enacyloxin increased modestly the GTPase activity of the wild type E. coli EF-Tu (less than 2 fold), introduction of single or multiple substitutions enhanced the catalytic activity, especially with the double (F46Y/Q48E) and the quadruple (A42E/A45K/F46Y) Q48E) substitutions where a 10 fold and a 25 fold stimulations were observed, respectively. These results suggest that amino acid substitutions in the "effector region" affect the catalytic GTPase activity of the E. coli factor.

\section{Discussion}

Frateuria sp. W-315 is a Gammaproteobacterium of the family Xanthomonadaceae. It is an intermediate strain among the genera Gluconobacter, Pseudomonas and Acetobacter [29]. It produces a family of polyenic antibiotics named enacyloxin active against Gram positive and Gram-negative microorganisms [6]. Among them enacyloxin Ila has been shown to inhibit bacterial protein biosynthesis in vitro [8]. Enacyloxin has a mechanism of action similar to that of the antibiotic kirromycin because it inhibits protein synthesis by acting on a specific target, the translation factor EF-Tu. Enacyloxin induces a constitutive activation of EF-Tu•GDP, making this complex so firmly attached to the mRNA-programmed ribosomes after aa-tRNA binding and GTP hydrolysis, that recycling of EF-Tu॰GDP and peptide bond formation are inhibited [9]. It is generally accepted that an organism that produces an antibiotic would not be sensitive to this molecule. Therefore, it is not surprising that the enacyloxin producing Frateuria W-315 strain is resistant to this antibiotic. However, the molecular mechanism of this resistance is not yet elucidated. In this context, taking into account the general increase in resistance toward antibiotics, and considering that the resistance of the enacyloxin producing Frateuria W-315 strain might stem from structural elements specific to EF-Tu, we have purified the factor from Frateuria W-315 and shown that it was as sensitive to enacyloxin as E. coli EF-Tu in in vitro poly(Phe) synthesis with a $\mathrm{IC}_{50}$ of $0.2 \mu \mathrm{M}$. The same results were obtained in the presence of enacyloxin Ila or in the presence of its intermediate biosynthesized form enacyloxin IVa, with S30 extracts of Frateuria W-315 suggesting that resistance to the antibiotic of enacyloxin producing Frateuria $\mathrm{W}-315$ is not linked to its target EFTu or to other component of the translational machinery. Therefore, it is most probable that the resistance to enacyloxin is due to an active secretion factor that removes quickly the antibiotic from the cytosol.

We can mention that none of the residues (K124, D316, T375) identified on E. coli EF-Tu responsible for enacyloxin resistance [16] are present in the amino acid sequence of Frateuria EF-Tu.

EF-Tu E. coli folds in three domains: the nucleotide-binding domain 1 (residues $1-199$ ) contains an $\alpha / \beta$ Rossman fold with four $\alpha$-helices A, B, C and D, as well as the "effector region" (residues 41-65), whereas domains 2 and 3 (residues 209-299 and $300-393$, respectively) are $\beta$-barrels. The binding site of Enacyloxin Ila located at the interface of domains 1 and 3 overlaps that of kirromycin [30]. On the basis of strong primary structure similarities between EF-Tu Frateuria and EF-Tu Ralstonia solanacearum, as revealed by mass spectrometric analyses, we have cloned and sequenced the gene coding for EF-Tu Frateuria. The amino acid sequence of the latter shows $80 \%$ identity with EF-Tu E. coli and $97 \%$ with EF-Tu Ralstonia. Only few differences occurred in fragment 36-48 belonging to the "effector region" and in fragment 182-186 located in domain 1 of the factor.

Inspite of these strong primary structure similarities, EF-Tu E. coli was shown to be more efficient than EF-Tu Frateuria in stimulating protein synthesis in vitro, suggesting that some key amino acid residues involved in catalysis might be different in the two factors. For example, when the Phe residue at position 46 or the Gln residue at position 48 in EF-Tu E. coli were changed to Tyr or Glu (the amino acid residues naturally present at these positions in EFTu Frateuria), respectively, the concentration inducing half maximal activation in poly(Phe) synthesis was not affected, suggesting that these substitutions operated in the "effector region" are not critical for the function of the factor. By contrast, changing Ala to Glu at position 42 and Ala to Lys at position 45 were shown to decrease the efficiency of EF-Tu E. coli. Taking into account the fact that Glu and Lys are charged residues and that their side chains are much larger than that of Ala, these results would be the reflect of structural requirements in the "effector region" of EF-Tu. The aforementioned effects were even more pronounced with the quadruple (A42E/A45K/F46Y/Q48E) substitutions, with a ka value of EF-Tu E. coli becoming almost equal to that of EF-Tu Frateuria. Altogether, these results suggest that the residues Glu- 42 , Lys-45, Tyr46 and Glu-48 participate to the binding pocket for the effector in the 3-D structure of EF-Tu Frateuria.

EF-Tu is the target of four families of antibiotics of unrelated structures inhibiting protein synthesis. Their prototypes are kirromycin, enacyloxin Ila, pulvomycin and GE2270A [10]. All these 

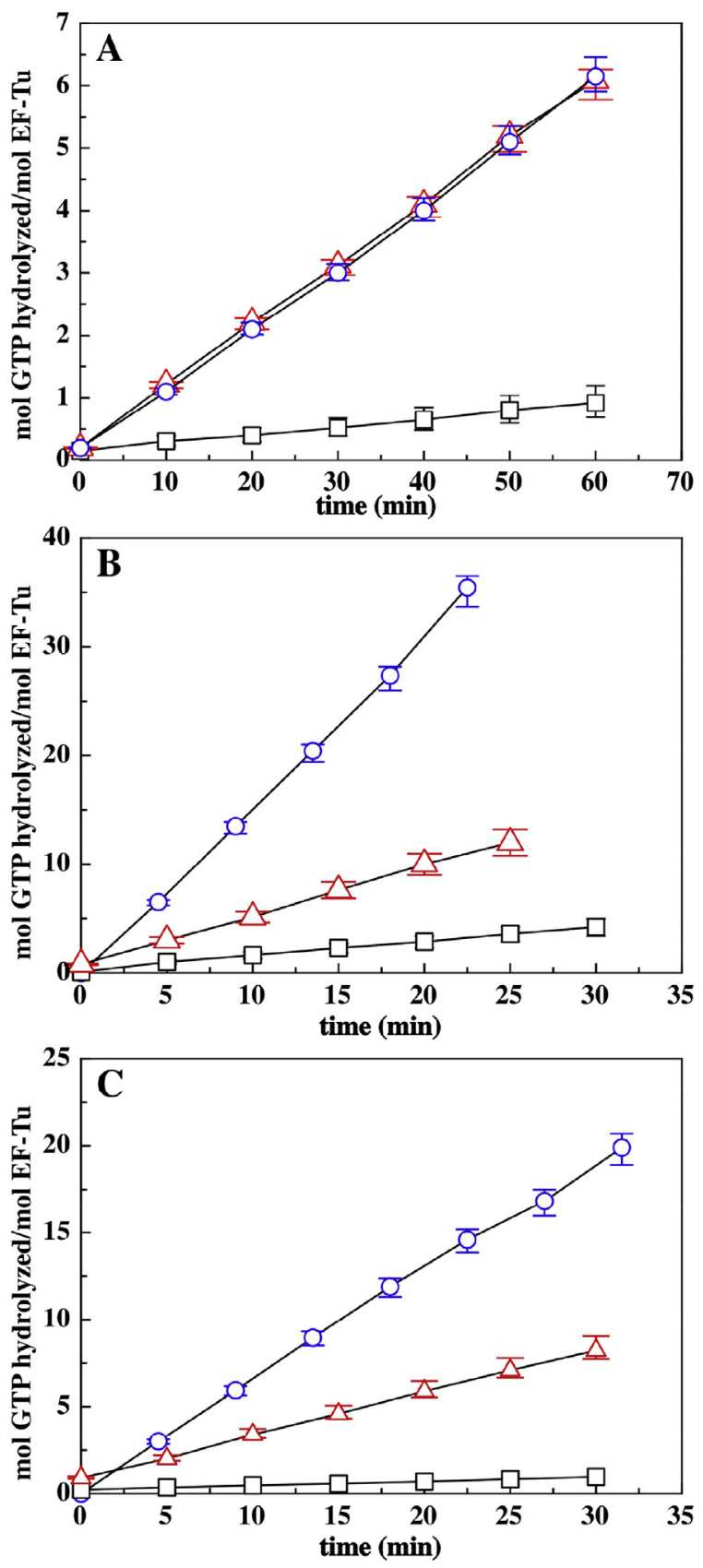

Fig. 6. Intrinsic GTPase activity of EF-Tu E. Coli $(\square)$, EF-Tu Frateuria W315 $(\triangle)$ and EFTu Coli A43E/A46K/F47Y/Q49E $(O)$ in the absence $(\mathrm{A})$ or in the presence of $50 \mu \mathrm{M}$ kirromycin (B) or $50 \mu \mathrm{M}$ enacyloxin IIa (C). The hydrolysis of $\left[\gamma^{32} \mathrm{P}\right] \bullet \mathrm{GTP}$ was determined as described in the methods. The concentration of preformed EF-Tu॰ $\left[\gamma-{ }^{32} \mathrm{P}\right] \bullet$ GTP complex was determined by nitrocellulose binding assay from the radioactivity bound to EF-Tu products in the presence of a saturating amount of $\left[\gamma-{ }^{32} \mathrm{P}\right] \bullet \mathrm{GTP}$ $\left(500 \mu \mathrm{M}-20 \mathrm{~Bq} \mathrm{pmol}^{-1}\right)$ in $40 \mathrm{mM}$ Tris- $\mathrm{HCl} \mathrm{pH} \mathrm{7.5,} 7 \mathrm{mM} \mathrm{MgCl}_{2}, 80 \mathrm{mM} \mathrm{NH}_{4} \mathrm{Cl}, 2 \mathrm{mM}$ Phosphoenolpyruvate, $100 \mu \mathrm{g} \mathrm{ml} \mathrm{m}^{-1}$ pyruvate Kinase, $1 \mathrm{mM}$ dithiothreitol, $0.05 \mathrm{mg} \mathrm{mL}^{-1} \mathrm{BSA}$ after $10 \mathrm{~min}$ at $30^{\circ} \mathrm{C}$

antibiotics strongly increase the affinity of EF-Tu•GTP by affecting the apparent association and dissociation rates in opposite ways, the association rate being strongly stimulated, while the dissociation rate is inhibited. The effect of these different antibiotics on the stimulation of the apparent GTP association rate is lower with EF-Tu Frateuria than with EF-Tu E. coli, while the effect on the dissociation rate is of the same order of magnitude. In the case of the antibiotic enacyloxin, the effect on the stimulation of the association rate was
8 times less important with EF-Tu Frateuria than with EF-Tu E. coli. With the exception of the antibiotic GE2270A, all the antibiotics exert a stronger effect on the stimulation of the apparent GDP dissociation rate of EF-Tu Frateuria in comparison with EF-Tu E. coli. These results would suggest that, inspite of the high degree of structural conservation, it exists some variations in the binding mode of the antibiotics on EF-Tu Frateuria and EF-Tu E. coli.

Conserved Thr-25 is involved in the coordination of the essential magnesium ion interacting with the $\beta$ - and $\gamma$-phosphates of the GTP nucleotide in the hydrophobic guanine binding pocket of the factor [31-34]. It is most probable that the modification of the effector loop structure in EF-Tu Frateuria results in a loss of hydrophobic contacts with the Thr-25 side chain methyl group that might affect the kinetics of interaction with the guanine nucleotides. Antibiotics bind at the interface between domains 1 and 3 of EF-Tu and destabilize the effector region by changing its conformation $[30,35]$. They contact the domain 1 interface representing a strategic region which communicates with the nucleotide binding pocket via the switch regions, and mediates the GDP and GTP dependent signals controlling the interactions with ligands.

In the present report, enacyloxin Ila was shown to enhance the intrinsic GTPase activity of EF-Tu E. coli, much more weakly than kirromycin. GTP hydrolysis in EF-Tu takes place through an in line direct attack on the $\gamma$-phosphate by a water molecule. His-84 has been proposed to be involved in EF-Tu-mediated GTPase activity through a nucleophilic attack of its side chain on the $\gamma$-phosphate $[11,31,36]$. However analysis of the GTPase activity of the EFTu•antibiotic complexes and of H84 mutants in the absence or in the presence of programmed ribosomes did not support a direct involvement of the H84 side chain in the GTP $\gamma$-phosphate hydrolysis $[10,37,38]$. All the antibiotics (kirromycin, enacyloxin, pulvomycin) that enhance the intrinsic GTPase activity of EF-Tu interact with the domain 1:3 interface. Perturbation of this area in close contact with the nucleotide binding pocket via the switch regions could influence the cleavage of the GTP $\gamma$-phosphate. GTPase activation is controlled by a hydrophobic gate formed by residues Val20 in the P-loop region and Ile-60 in the switch I effector region which prevents His-84 from activating the water molecule and the catalysis of GTP hydrolysis.

Switch 1 region (residues 40-62) displays conformational changes in the working cycle of EF-Tu. The prominent structural differences between EF-Tu E. coli and EFTu Frateuria are located in the switch 1 region. This structural modification in switch 1 in EFTu Frateuria can affect the flexibility of this region and facilitate the opening gate and the access of His-84 to the nucleotide. As a consequence, the GTPase activity of EF-Tu Frateuria might be increased in comparison with that of EF-Tu E. coli. In line with this, mutations in this region that replaced residues in EF-Tu E. coli by those of EF-Tu Frateuria activate intrinsic GTP hydrolysis and antibiotics dependent stimulation. Crystallographic studies of EF-Tu Frateuria in complex with the non hydrolysable GTP and/or with antibiotics will bring further insight into the GTPase mechanism.

\section{Acknowledgments}

We are indebted to Dr T. Watanabe and colleagues for the gift of the strain Frateuria sp.W-315. We gratefully thank A. Parmeggiani and S. Baouz for suggestions and for critical reading of the manuscript.

\section{References}

[1] D.N. Wilson, K.H. Nierhaus, The ribosome through the looking glass, Angew. Chem. Int. Ed. Engl. 42 (2003) 3464-3486.

[2] J. Nilsson, P. Nissen, Elongation factors on the ribosome, Curr. Opin. Struct. 
Biol. 15 (2005) 349-354.

[3] D. Kavaliauskas, P. Nissen, C.R. Knudsen, The busiest of all ribosomal assistants: elongation factor Tu, Biochemistry 51 (2012) 2642-2651.

[4] A. Bashan, I. Agmon, R. Zarivach, F. Schluenzen, J. Harms, R. Berisio, H. Bartels F. Franceschi, T. Auerbach, H.A. Hansen, E. Kossoy, M. Kessler, A. Yonath, Structural basis of the ribosomal machinery for peptide bond formation, translocation, and nascent chain progression, Mol. Cell 11 (2003) 91-102.

[5] A. Parmeggiani, P. Nissen, Elongation factor Tu-targeted antibiotics: four different structures, two mechanisms of action, FEBS Lett. 580 (2006) 4576-4581.

[6] T. Watanabe, K. Izaki, H. Takahashi, New polyenic antibiotics active against gram-positive and -negative bacteria. I. Isolation and purification of antibiotics produced by Gluconobacter sp. W-315, J. Antibiot. (Tokyo) 35 (1982) $1141-1147$.

[7] T. Watanabe, N. Okubo, T. Suzuki, K. Izaki, New polyenic antibiotics active against gram-positive and gram-negative bacteria. VI. Non-lactonic polyene antibiotic, enacyloxin Ila, inhibits binding of aminoacyl-tRNA to A site of ribosomes, J. Antibiot. (Tokyo) 45 (1992) 572-574.

[8] T. Watanabe, T. Suzuki, K. Izaki, New polyenic antibiotics active against grampositive and gram-negative bacteria. V. Mode of action of enacyloxin Ila, J. Antibiot. (Tokyo) 44 (1991) 1457-1459.

[9] R. Cetin, I.M. Krab, P.H. Anborgh, R.H. Cool, T. Watanabe, T. Sugiyama, K. Izaki, A. Parmeggiani, Enacyloxin Ila, an inhibitor of protein biosynthesis that acts on elongation factor Tu and the ribosome, EMBO J. 15 (1996) 2604-2611.

[10] I.M. Krab, A. Parmeggiani, Mechanisms of EF-Tu, a pioneer GTPase, Prog. Nucleic Acid. Res. Mol. Biol. 71 (2002) 513-551.

[11] T. Hogg, J.R. Mesters, R. Hilgenfeld, Inhibitory mechanisms of antibiotics targeting elongation factor Tu, Curr. Protein Pept. Sci. 3 (2002) 121-131.

[12] H. Wolf, D. Assmann, E. Fischer, Pulvomycin, an inhibitor of protein biosynthesis preventing ternary complex formation between elongation factor Tu, GTP, and aminoacyl-tRNA, Proc. Natl. Acad. Sci. U. S. A. 75 (1978) 5324-5328.

[13] P.H. Anborgh, A. Parmeggiani, New antibiotic that acts specifically on the GTPbound form of elongation factor Tu, EMBO J. 10 (1991) 779-784.

[14] P.H. Anborgh, S. Okamura, A. Parmeggiani, Effects of the antibiotic pulvomycin on the elongation factor Tu-dependent reactions. Comparison with other antibiotics, Biochemistry 43 (2004) 15550-15556.

[15] A. Parmeggiani, I.M. Krab, S. Okamura, R.C. Nielsen, J. Nyborg, P. Nissen, Structural basis of the action of pulvomycin and GE2270 A on elongation factor Tu, Biochemistry 45 (2006) 6846-6857.

[16] A.M. Zuurmond, L.N. Olsthoorn-Tieleman, J. Martien de Graaf, A. Parmeggiani, B. Kraal, Mutant EF-Tu species reveal novel features of the enacyloxin IIa inhibition mechanism on the ribosome, J. Mol. Biol. 294 (1999) 627-637.

[17] M. Salanoubat, S. Genin, F. Artiguenave, J. Gouzy, S. Mangenot, M. Arlat, A. Billault, P. Brottier, J.C. Camus, L. Cattolico, M. Chandler, N. Choisne, C. Claudel-Renard, S. Cunnac, N. Demange, C. Gaspin, M. Lavie, A. Moisan, C. Robert, W. Saurin, T. Schiex, P. Siguier, P. Thebault, M. Whalen, P. Wincker, M. Levy, J. Weissenbach, C.A. Boucher, Genome sequence of the plant pathogen Ralstonia solanacearum, Nature 415 (2002) 497-502.

[18] I.M. Krab, A. Parmeggiani, Functional-structural analysis of threonine 25, a residue coordinating the nucleotide-bound magnesium in elongation factor Tu, J. Biol. Chem. 274 (1999) 11132-11138.

[19] P.H. Anborgh, A. Parmeggiani, Probing the reactivity of the GTP- and GDPbound conformations of elongation factor Tu in complex with the antibiotic GE2270 A, J. Biol. Chem. 268 (1993) 24622-24628.

[20] R.J. Smith, D.H. Williams, J.C.J. Barna, I.R. McDermott, K.D. Haegele, F. Piriou, J. Wagner, W. Higgins, Structure revision of the antibiotic Pulvomycin, J. Am.
Chem. Soc. 107 (1985) 2849-2857.

[21] G. Chinali, A. Parmeggiani, Properties of the elongation factors from Escherichia coli. Exchange of elongation factor $\mathrm{G}$ during elongation of polypeptide chain, Eur. J. Biochem. 32 (1973) 463-472.

[22] I.M. Krab, R. te Biesebeke, A. Bernardi, A. Parmeggiani, Elongation factor Ts can act as a steric chaperone by increasing the solubility of nucleotide bindingimpaired elongation factor-Tu, Biochemistry 40 (2001) 8531-8535.

[23] J.B. Crechet, D. Canceill, V. Bocchini, A. Parmeggiani, Characterization of the elongation factors from calf brain. 1. Purification, molecular and immunological properties, Eur. J. Biochem. 161 (1986) 635-645.

[24] O. Fasano, W. Bruns, J.B. Crechet, G. Sander, A. Parmeggiani, Modification of elongation-factor-Tu. guanine-nucleotide interaction by kirromycin. A comparison with the effect of aminoacyl-tRNA and elongation factor Ts, Eur. J. Biochem. 89 (1978) 557-565.

[25] R. Oyama, T. Watanabe, H. Hanzawa, T. Sano, T. Sugiyama, K. Izaki, An extracellular Quinoprotein oxidase that catalyses conversion of Enacyloxin IVa to Enacyloxin IIa, Biosci. Biotechnol. Biochem. 58 (1994) 1914-1917.

[26] H. Wolf, G. Chinali, A. Parmeggiani, Mechanism of the inhibition of protein synthesis by kirromycin. Role of elongation factor Tu and ribosomes, Eur. J. Biochem. 75 (1977) 67-75.

[27] D. Picone, A. Parmeggiani, Transfer ribonucleic acid deprived of the C-C-A 3'extremity can interact with elongation factor Tu, Biochemistry 22 (1983) 4400-4405.

[28] O. Fasano, J.B. Crechet, A. Parmeggiani, Preparation of nucleotide-free elongation factor Tu and its stabilization by the antibiotic kirromycin, Anal. Biochem. 124 (1982) 53-58.

[29] T. Watanabe, T. Sugiyama, K. Izaki, New polyenic antibiotics active against gram-positive and gram-negative bacteria. IX. Reclassification of a strain W315 producing enacyloxins, J. Antibiot. (Tokyo) 47 (1994) 496-498.

[30] A. Parmeggiani, I.M. Krab, T. Watanabe, R.C. Nielsen, C. Dahlberg, J. Nyborg, P. Nissen, Enacyloxin Ila pinpoints a binding pocket of elongation factor Tu for development of novel antibiotics, J. Biol. Chem. 281 (2006) 2893-2900.

[31] H. Berchtold, L. Reshetnikova, C.O. Reiser, N.K. Schirmer, M. Sprinzl, R. Hilgenfeld, Crystal structure of active elongation factor Tu reveals major domain rearrangements, Nature 365 (1993) 126-132.

[32] M. Kjeldgaard, P. Nissen, S. Thirup, J. Nyborg, The crystal structure of elongation factor EF-Tu from Thermus aquaticus in the GTP conformation, Structure 1 (1993) 35-50.

[33] K. Abel, M.D. Yoder, R. Hilgenfeld, F. Jurnak, An alpha to beta conformational switch in EF-Tu, Structure 4 (1996) 1153-1159.

[34] G. Polekhina, S. Thirup, M. Kjeldgaard, P. Nissen, C. Lippmann, J. Nyborg, Helix unwinding in the effector region of elongation factor EF-Tu-GDP, Structure 4 (1996) 1141-1151.

[35] L. Vogeley, G.J. Palm, J.R. Mesters, R. Hilgenfeld, Conformational change of elongation factor Tu (EF-Tu) induced by antibiotic binding. Crystal structure of the complex between EF-Tu.GDP and aurodox, J. Biol. Chem. 276 (2001) $17149-17155$

[36] T. Daviter, H.J. Wieden, M.V. Rodnina, Essential role of histidine 84 in elongation factor Tu for the chemical step of GTP hydrolysis on the ribosome, J. Mol. Biol. 332 (2003) 689-699.

[37] R.H. Cool, A. Parmeggiani, Substitution of histidine-84 and the GTPase mechanism of elongation factor Tu, Biochemistry 30 (1991) 362-366.

[38] G. Scarano, I.M. Krab, V. Bocchini, A. Parmeggiani, Relevance of histidine-84 in the elongation factor Tu GTPase activity and in poly(Phe) synthesis: its substitution by glutamine and alanine, FEBS Lett. 365 (1995) 214-218. 